\title{
Análise de crateras lunares como ferramenta alternativa para o ensino de física e astronomia
}

\author{
Lunar crater analysis as an alternative tool for physics and astronomy teaching
}

\author{
Ibson José Maciel Leite ${ }^{2}$, Cícero Jailton de Morais Souza ${ }^{2,3}$, Allan Christopher Rodrigues Barboza ${ }^{2}$, \\ José Weslley Pereira da Silva², Mário Anonio Alves Monteiro ${ }^{* 1,2}$ [] \\ ${ }^{1}$ Instituto Federal de Educação, Ciência e Tecnologia de Pernambuco, Recife, PE, Brasil \\ ${ }^{2}$ Instituto Federal de Educação, Ciência e Tecnologia de Pernambuco, Física Pesqueira, PE, Brasil \\ ${ }^{3}$ Instituto Federal de Educação, Ciência e Tecnologia do Sertão Pernambucano, Salgueiro, PE, Brasil
}

\begin{abstract}
Recebido em 02 de Julho de 2019. Revisado em 26 de Dezembro de 2019. Aceito em 23 de Janeiro de 2020.
Neste trabalho realizamos medições indiretas dos tamanhos de algumas crateras lunares, bem como, de suas coordenadas selenográficas a partir de fotografias da superfície lunar. Para realizar as análises das imagens e as medições desenvolvemos um software em Visual Basic, que denominamos Îasy. Comparando os resultados obtidos por meio do programa de computador desenvolvido com dados disponibilizados por um observatório astronômico, obtivemos desvios percentuais médios menores que 1\%. Diante do potencial didático da ferramenta, capaz de reduzir as abstrações e produzir motivação, assim como, da confiabilidade dos resultados obtidos, constatamos que o software Îasy pode ser usado como ferramenta educacional, além de auxiliar astrônomos amadores no desenvolvimento de pesquisas e medições com boa precisão.
\end{abstract}

Palavras-chave: crateras lunares, coordenadas selenográficas, informática e astronomia, ensino de astronomia.

In this paper indirect measurements of the sizes of some lunar craters as well as their selenographic coordinates from lunar surface photographs were performed. In order to carry out the analysis of the images a software was developed in Visual Basic, which is so called Îasy. Comparing the results obtained through the program developed with data provided by an astronomical observatory average percentage deviations of less than $1 \%$ were obtained. In view of the didactic potential of the tool capable of reducing abstractions and producing motivation, as well as the reliability of the obtained results, we can verify that Îasy software can be used as an educational tool, as well as assist amateur astronomers in the development of good precision.

Keywords: lunar craters, selenographic coordinates, computer science and astronomy, astronomy teaching.

\section{Introdução}

A Lua tem fascinado a humanidade por muito tempo, com suas fases, seus eclipses, sua importância na marcação do tempo e suas crateras que, quando vistas a olho nu, parecem "manchas" que em algumas culturas foram associadas a figuras mitológicas. Um dos primeiros a tentar encontrar as dimensões da Lua foi o grego Aristarco de Samos, nascido por volta de 310 a. E. C. em uma das ilhas jônicas. Por meio da observação de eclipses lunares e solares, Aristarco fez uma comparação entre os diâmetros da Lua, da Terra e do Sol. A sua única obra que sobreviveu ao tempo foi: "Sobre os tamanhos e a distância do Sol e da Lua" [1].

Em 1609, Galileu Galilei, o primeiro homem a observar a Lua por meio de um telescópio, percebeu em suas observações que ela tinha crateras, relevos e montanhas. Segundo Nussenzveig [2], ao observar a Lua, Galileu verificou que:

*Endereço de correspondência: mariomonteiro@pesqueira.ifpe.edu.br
Não era uma esfera perfeita como pretendiam os aristotélicos, mas tinha vales profundos e cadeias de montanhas elevadas, cuja altura conseguiu estimar, a partir da sombra projetada pelos raios solares, como sendo comparável à das montanhas terrestres.

A utilização do telescópio foi um marco para a observação de detalhes dos astros, características que até então eram imperceptíveis. Utilizando sua luneta, Galileu pôde observar a Lua com um aumento nunca visto na época. No livro intitulado Sidereus Nuncius: O Mensageiro das Estrelas, Galileu expõe desenhos que fez da superfície lunar com base em suas observações telescópicas. A Fig. 1 apresenta alguns destes desenhos. Além de observar e desenhar o relevo da superfície lunar, Galileu tentou também determinar o tamanho das "proeminências" e "depressões" lunares:

Parece-me ter ficado suficientemente claro, pelas aparências já explicadas, que a superfície mais brilhante da Lua esteja coberta 


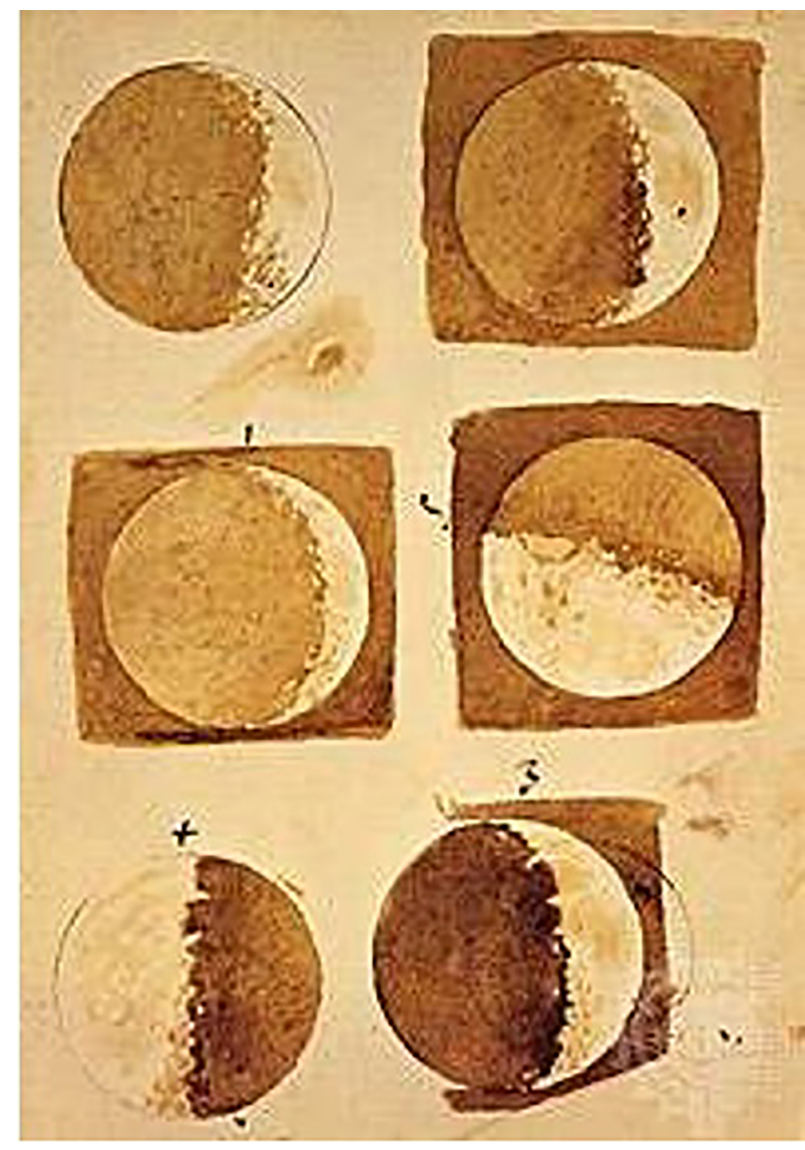

Figura 1: Desenhos de Galileu Galilei da Lua, conforme ele a via com sua luneta, entre 1609/1610. Fonte: Grupo de Estudos e Pesquisas "História, Sociedade e Educação no Brasil", UNICAMP

por todo o lado com proeminências e depressões. Falta-nos agora falar acerca dos seus tamanhos, demonstrando que as rugosidades terrestres são muito menores do que as lunares; digo menores falando absolutamente, não apenas em proporção aos tamanhos dos seus globos [3].

A partir da última metade do século XX começaram a ser desenvolvidos softwares especializados em realizar estimativas das dimensões dos astros e outras características deles, utilizando teoremas matemáticos aplicados à programação computacional e análise de imagens digitalizadas. Em um estudo pioneiro publicado em 1964, Robert Wildey criou um programa escrito na linguagem Fortran para transformar coordenadas celestes (ascensão reta, declinação e tempo universal) de crateras lunares em coordenadas selenográficas [4]. Já em um trabalho mais recente, Kneissl, Gasselt e Neukumet [5], desenvolveram uma extensão de software para o ArcMap, um componente de um conjunto de programas especializados em processamentos geoespaciais da Environmental Systems Research Institute's (ESRI's). Esta extensão permite que o usuário realize medidas dos tamanhos de crateras em superfícies planetárias, independentemente das distorções nas imagens causadas pelo distanciamento das crateras do centro do disco planetário. Quando aplicados à Lua, estes tipos de softwares tornam mais prático e eficiente a determinação das dimensões do nosso satélite natural. Por isso, tais ferramentas tornaram-se indispensáveis para os modernos estudos de Astronomia e Astrofísica, auxiliando na construção de modelos mais precisos dos fenômenos naturais.

Neste trabalho desenvolvemos o software Îasy (que significa Lua em Tupi), cujo objetivo é analisar e medir as dimensões das crateras da Lua e suas respectivas coordenadas selenográficas, visando proporcionar uma ferramenta prática e sem custos com licenças, capaz de auxiliar astrônomos amadores em medições e docentes de física e ciências em geral com uma ferramenta educacional. Este software foi construído na plataforma Microsoft Visual Basic 6.0, que é uma linguagem de programação orientada a objetos, introduzida pela Microsoft em 1998, e por seus diversos recursos foi bastante utilizada em ambientes corporativos no desenvolvimento de programas comerciais. Utilizamos esta linguagem devido a sua grande versatilidade e relativa facilidade em comparação com outras ferramentas de desenvolvimento computacional.

\section{Crateras lunares e os ensinos de física e astronomia}

Conforme a Base Nacional Comum Curricular (BNCC), a área de Ciências da Natureza e suas Tecnologias para o Ensino Médio, articulada com a área de Ciências da Natureza do Ensino Fundamental, deve permitir ao aluno desenvolver determinadas competências e habilidades específicas. Dentre as competências apontadas pela BNCC, destacamos: (1) analisar e utilizar de interpretações sobre a dinâmica da Terra e do Cosmos para elaborar argumentos e realizar previsões sobre o funcionamento e a evolução do Universo; e (2) "investigar situaçõesproblema e avaliar aplicações do conhecimento científico e tecnológico e suas implicações no mundo, utilizando procedimentos e linguagens próprios das Ciências da Natureza" [6].

O estudo das crateras lunares, suas dimensões e formações, pode ser incluído nestas competências, uma vez que podemos abordar este tema para compreender a dinâmica de origem e evolução dos corpos celestes que fazem parte do sistema solar. Essa discussão pode ser realizada a partir de situações-problema levantadas pelos docentes, e resolvidas utilizando as ferramentas científicas adequadas. Assim, é necessário apresentar aos estudantes os "procedimentos e instrumentos de investigação" [6] da Ciência, como a identificação de problemas, a formulação de questões, o teste de hipóteses, a elaboração de argumentos e explicações, e a escolha e utilização de instrumentos de medida adequados. Neste sentido, 
é importante, como destaca a BNCC, que o estudante desenvolva as habilidades de:

Construir questões, elaborar hipóteses, previsões e estimativas, empregar instrumentos de medição e representar e interpretar modelos explicativos, dados e/ou resultados experimentais para construir, avaliar e justificar conclusões no enfrentamento de situaçõesproblema sob uma perspectiva científica [6].

Para que tais habilidades e competências sejam desenvolvidas pelos alunos é imprescindível que, junto ao pensamento científico, sejam introduzidas às relações matemáticas utilizadas para descrever a realidade física na qual estão inseridos, e as ferramentas tecnológicas que irão possibilitar a integração entre estes fenômenos celestes, tão distantes do ponto de vista espacial, e os objetos, situações e fenômenos que fazem parte do cotidiano do aluno.

É neste contexto que discutimos neste artigo o estudo das crateras lunares na educação. Este tema não é algo inédito no ensino de Astronomia. Como apontado por Langhi [7], é possível estudar a formação de crateras de forma prática, através do impacto, com gesso ou areia, de esferas de diversos materiais. Outra possibilidade levantada pelo autor é observar a Lua por algumas horas através de um telescópio, onde "é possível notar a 'linha' divisória do dia/noite lunar mover-se sensivelmente sobre as crateras na superfície da Lua, provocando um aumento (ou diminuição) da parte iluminada" [7].

Seguindo essa mesma linha de pensamento, Scott et al. [8] propõem a introdução de tópicos de ciência planetária, especificamente a formação de crateras, no ensino, com a justificativa de que tal atividade é uma oportunidade para que os alunos obtenham um aprendizado através da participação em uma investigação científica relevante para o mundo real. Os estudantes tinham que medir a profundidade de uma cratera de impacto usando o comprimento das sombras e o ângulo dos raios de luz de uma fonte que iluminava as mesmas.

Também é necessário levar em consideração que a Astronomia não é ensinada apenas em espaços formais de ensino. Existem diversas instituições no Brasil e no mundo que trabalham para despertar o interesse e a curiosidade por essa ciência, impactando de forma direta no estímulo da aprendizagem de seus conceitos. Para Langhi e Martins [9],

A responsabilidade de alfabetizar a comunidade não fica somente restrita à escola, uma vez que espaços não escolares também possuem uma característica que pode contemplar a aprendizagem: a característica da motivação intrínseca. Desta forma, o potencial do papel da educação não escolar em contribuir para a aprendizagem precisa ser aproveitado, pois pode oferecer o que muitas escolas não apresentam.
Um exemplo de uma relevante iniciativa científica voltada para pesquisa, educação e divulgação da Astronomia, foi o projeto online Moon Zoo, desenvolvido pela Citizen Science Alliance, criado em maio de 2010 e encerrado em junho de 2015. Neste projeto, usuários registrados no site tinham a tarefa de, dentre outras coisas, identificar e medir crateras lunares por meio de uma interface gráfica especialmente projetada [10]. Através de imagens de alta resolução disponibilizadas pela National Aeronautics and Space Administration (NASA), usuários em qualquer lugar do mundo, conectados à internet, podiam explorar a superfície lunar, o que acabou gerando uma enorme quantidade de dados provenientes de múltiplas observações diferentes. Dentre os principais objetivos do projeto, estava catalogar a localização e as dimensões de pequenas crateras de impacto, o que permitiria determinar as idades das crateras com bastante precisão:

Como as dimensões de uma determinada cratera são avaliadas por vários usuários no Moon Zoo, a variabilidade estatística dessas localizações de elipse/círculo pode ser analisada e explorada para determinar o estado da degradação da cratera, que fornece [uma] estimativa independente da idade da superfície [10].

Em uma pesquisa publicada em 2013, PRATHER et al. [11] analisaram o desempenho dos voluntários (também chamados de "cidadãos cientistas") do projeto Moon Zoo em determinadas tarefas. O principal objetivo da pesquisa era aferir, utilizando-se instrumentos avaliativos especialmente desenvolvidos, o conhecimento conceitual e as habilidades de raciocínio, relacionados ao programa, dos "cidadãos cientistas" que participaram do projeto. Constatou-se que os voluntários que realizavam mais tarefas no Moon Zoo também eram aqueles que obtinham os melhores resultados nos instrumentos de avaliação propostos. Além disso, a pesquisa demonstrou que os principais motivos que estimularam essas pessoas a participarem do projeto eram: o interesse em Astronomia, o desejo de participar de uma pesquisa científica original e o deslumbramento com a imensidão do Universo.

\section{Diâmetro das crateras lunares}

O programa Îsy consegue estimar as dimensões das crateras lunares fazendo a análise de uma fotografia da Lua. Primeiramente, o programa obtém o raio da Lua em pixels, a partir de onde se pode fazer uma relação com o mesmo raio em quilômetros: $1.738 \mathrm{~km}$ [12], e assim estimar o diâmetro das crateras também em quilômetros.

Para isso, não é necessário que a fotografia seja da Lua inteira nem que apareça o seu centro, mas que mostre pelo menos parte de sua borda, de modo que seja possível marcar três pontos sobre ela. A Fig. 2 apresenta a interface do software Iasy. 


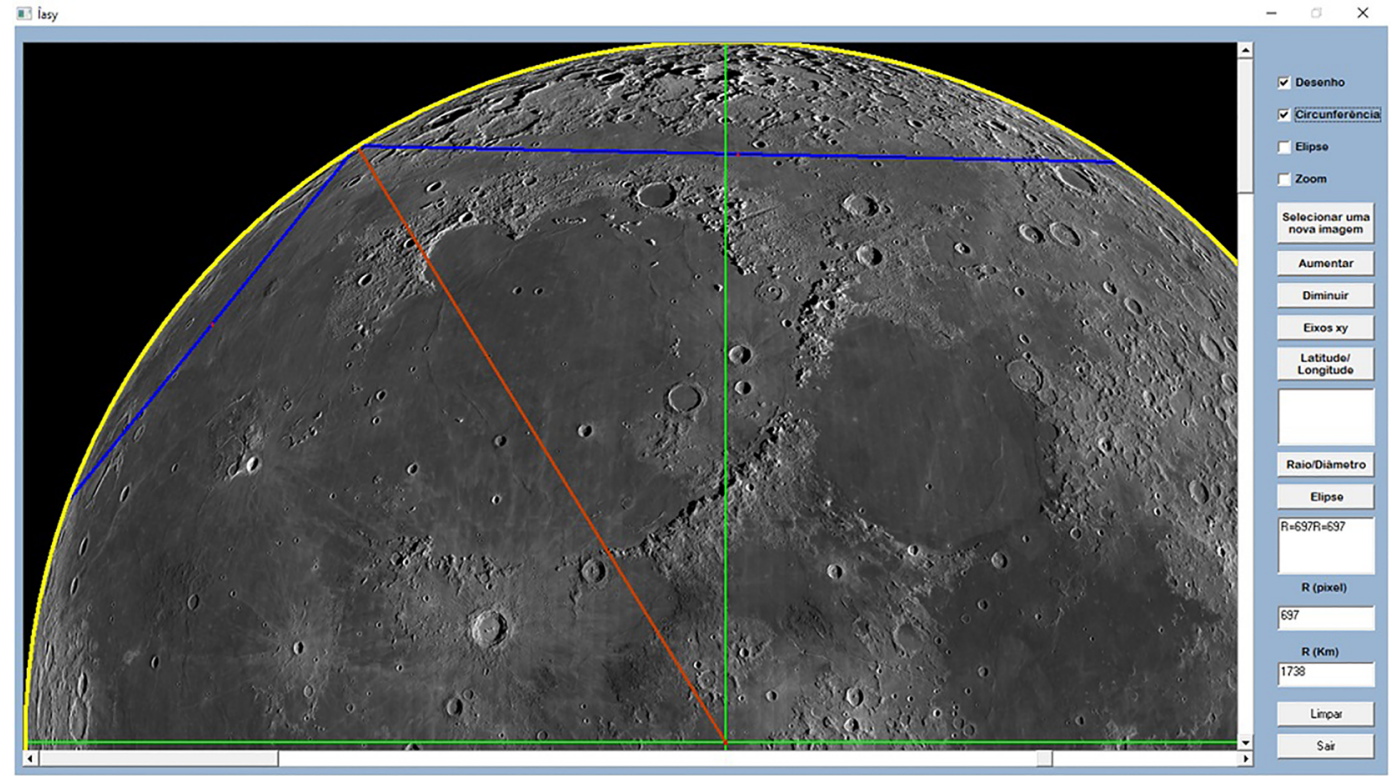

Figura 2: Interface do software Îasy. Fonte: Captura de tela do programa Îasy.

Para desenhar a circunferência que delimita o disco lunar, o usuário deve primeiramente deixar marcadas as opções "Desenho" e "Circunferência", clicar em três pontos sobre a borda da Lua e em seguida clicar em "Raio/Diâmetro". O resultado é o que se observa na Fig. 2 , onde as linhas verdes são os eixos $x$ e $y$ (que podem ser desenhados clicando-se em "Eixos xy"); as linhas azuis são os segmentos de retas que ligam os pontos selecionados; a linha vermelha é o segmento que une o segundo ponto selecionado e o centro da circunferência, calculado pelo programa; e a linha amarela é a circunferência traçada pelo T̂sy sobre a borda da Lua.

Para obter o raio da circunferência da Lua, o algoritmo do Îasy utiliza como ferramenta matemática o conceito de triângulos inscritíveis. Os três pontos aleatórios que devem ser marcados na borda da imagem da Lua são: P0 (X0, Y0), P1 (X1, Y1) e P2 (X2, Y2), que podem ser observados na Fig. 3.

Podemos traçar um segmento de reta ligando os pontos $P 0$ e $P 1$, e outro ligando os pontos $P 0$ e $P 2$. Chamaremos de $r_{1}$ (Reta 01) e $r_{2}$ (Reta 02), respectivamente, as retas suportes dos segmentos $\overline{P 0 P 1}$ e $\overline{P 0 P 2}$. Utilizando a equação (1) a seguir, é possível encontrar os coeficientes angulares, $m r_{1}$ e $m r_{2}$, das retas $r_{1}$ e $r_{2}$.

$$
m r_{i}=\frac{\Delta Y_{i}}{\Delta X_{i}}
$$

A partir da equação (2) abaixo, podemos determinar as inclinações $m s 1$ e $m s 2$ das mediatrizes dos segmentos $\overline{P 0 P 1}$ e $\overline{P 0 P 2}$

$$
m s_{i}=\frac{-\Delta Y_{i}}{\Delta X_{i}}
$$

Nas equações acima, $i$ é o índice relativo a cada segmento de reta e $\Delta X_{i}$ e $\Delta Y_{i}$ são definidos como indicado

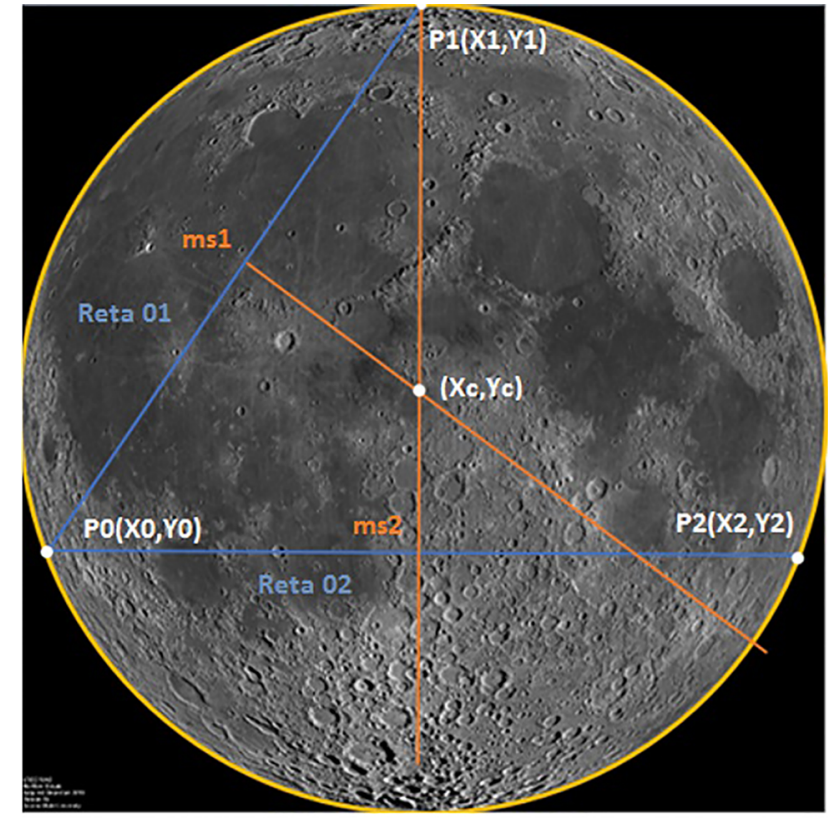

Figura 3: Esquema da obtenção do circuncentro da Lua, aproximada como uma circunferência. Fonte: Captura de tela do programa l̂asy.

a seguir:

$$
\begin{array}{rr}
\Delta X_{1}=(X 1-X 0) & \Delta X_{2}=(X 2-X 1) \\
\Delta Y_{1}=(Y 1-Y 0) & \Delta Y_{2}=(Y 2-Y 1)
\end{array}
$$

Segundo Costa et al. [13]: "As mediatrizes dos lados de um triângulo interceptam-se em um mesmo ponto (...), então, ele é centro de uma circunferência que circunscreve o triângulo (...)". Portanto, o ponto $\left(X_{C}, Y_{C}\right)$, que representa o cruzamento das mediatrizes de inclinações $m s_{1}$ e $m s_{2}$, é o centro da circunferência lunar. 
Uma vez obtido o centro da circunferência, estabelecemos a origem do nosso sistema de coordenadas no ponto $\left(X_{C}, Y_{C}\right)$. O raio $R$ (raio da Lua) pode ser determinado através da equação da distância entre dois pontos num plano cartesiano, sendo um desses pontos o centro da Lua e o outro um ponto qualquer sobre a borda do disco lunar de coordenadas $(X 1, Y 1)$ :

$$
R=\sqrt{(X 1-X C)^{2}+(Y 1-Y C)^{2}}
$$

Para conseguir estimar os diâmetros das crateras foi utilizado o conceito de elipse, pois as crateras sobre a superfície aproximadamente esférica da Lua apresentamse para nós, aqui na Terra, no formato elíptico, como podemos ver na Fig. 4.

As crateras com formas aproximadamente circulares também podem ser analisadas com a mesma ferramenta.

Depois de determinar o raio da Lua, deve-se clicar em "Limpar" e marcar "Elipse". O desenho da figura que circundará a borda da cratera analisada exige do operador do software um pouco de habilidade para que a elipse se adapte o mais precisamente possível ao formato da cratera, conforme visto na Fig. 4. A curva desenhada nesta imagem foi construída selecionando-se inicialmente dois pontos sobre a borda da depressão E1 e E2 respectivamente, de forma que estes devem coincidir com os extremos opostos do semieixo maior. Feito isto e após clicar em "Raio/Diâmetro", o programa nos fornece o diâmetro da cratera e uma mediatriz (linha tracejada na Fig. 4). Na intersecção entre o segmento de reta que liga E1, E2 e a mediatriz, devemos marcar obrigatoriamente o ponto $E 4$. O ponto $E 3$ deve ser inserido na intersecção entre a borda da cratera e a mediatriz. Dessa forma, o semieixo menor é definido como o segmento de reta que une E3 a E4. Ao clicar em "Elipse" o programa desenha a elipse vermelha, conforme a Fig. 4.

Partindo de interpretações geométricas e com o auxílio do software $\hat{I} a s y$, obtemos o semieixo maior $a$ e o menor

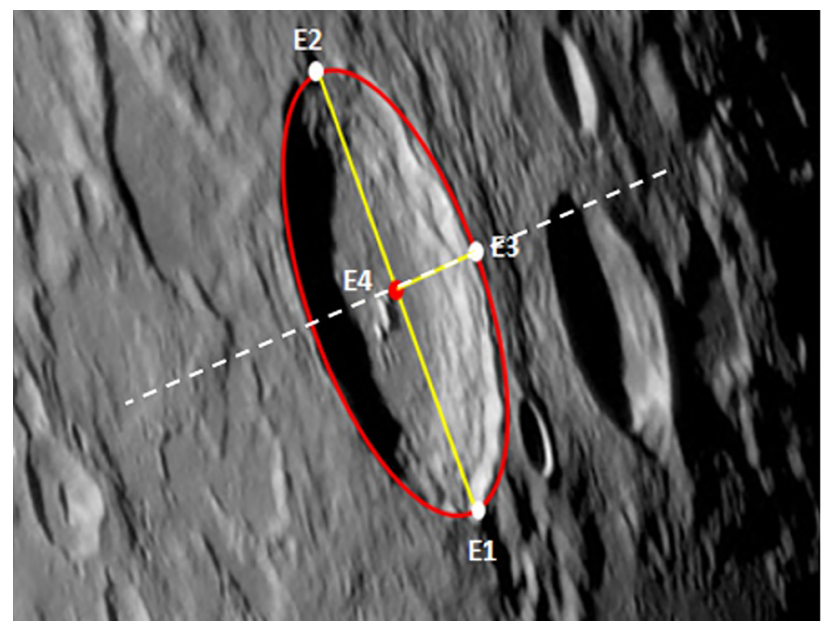

Figura 4: Software Îasy desenhando a elipse sobre a cratera Geminus. Fonte: Rubens Núcleo de Estudos da Lua , modificada pelo programa Îasy. $b$ da elipse, medindo, respectivamente, a distância entre os pontos E1 e $E_{4}$, e $E 3$ e $E_{4}$

Para determinarmos o diâmetro de uma cratera de formato aproximadamente circular, é necessário lembrar que a mesma depressão vista de uma posição diferente pode se apresentar como elipse. A Fig. 5 nos mostra que sua forma só é afetada na direção do semieixo menor, enquanto no semieixo maior não houve alteração significativa Logo, podemos concluir que o diâmetro da cratera é igual a duas vezes o comprimento do seu semieixo maior, conforme a equação (4).

$$
d=2 a
$$

Em crateras cujo formato aparente é circular, o diâmetro é o dobro do seu raio, como mostrado na equação (5).

$$
d=2 R
$$

Para descobrir as coordenadas do centro da cratera, utilizamos a equação da elipse:

$$
y=\frac{b}{a} \sqrt{a^{2}+x^{2}}
$$

Onde $a$ e $b$ são novamente os semieixos maior e menor, e $x$ e $y$ são as coordenadas do centro da elipse no plano lunar. Como previamente estabelecido o centro da elipse é o ponto E4 da Fig. 4, ou seja, a intersecção entre o segmento de reta que representa o eixo maior e sua mediatriz

\section{Coordenadas selenográficas (latitude e longitude lunar)}

Além da determinação do diâmetro das crateras lunares o software Îasy é capaz de obter um valor aproximado para as coordenadas selenográficas de cada cratera na superfície da Lua. Para isso, depois de determinar o diâmetro da cratera, basta clicar em "Latitude/Longitude"

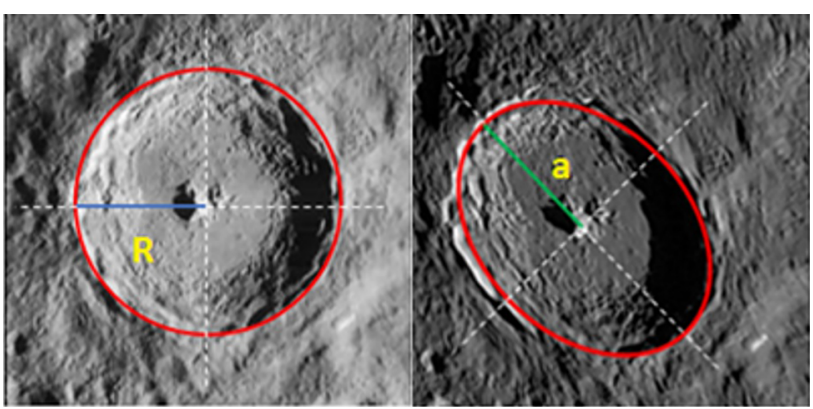

Figura 5: Fotografia da cratera Tycho Brahe, obtida por um satélite orbitando a Lua (imagem à esquerda) e fotografia da mesma cratera obtida da Terra (imagem à direita). Fonte: RUBENS NÚCLEO DE ESTUDOS DA LUA. Disponível em: <http://gaea-rubens.blogspot.com/2012/11/novasinterpretacoes-sobre-conhecida.html $>$. Acessado em: 06 de novembro de 2019 . 
e o programa irá fornecer a latitude e a longitude da cratera.

Como a imagem da Lua que consideramos é uma fotografia, ou seja, é uma representação em duas dimensões de um objeto tridimensional torna-se necessário promover uma transformação de sistemas de coordenadas. Para isso, utilizaremos o modelo matemático da Fig. 6, no qual a Lua está representada pela aproximação de uma esfera de raio $R$.

Definimos que o plano onde está a foto da Lua é o plano yz. Definimos também a origem do sistema de coordenadas como sendo o centro do círculo lunar.

Considerando o modelo da Fig. 6 e transformando as coordenadas cartesianas em esféricas temos que:

$$
\begin{gathered}
x=R \operatorname{sen}\left(\phi_{2}\right) \cos (\theta) \\
y=R \operatorname{sen}\left(\phi_{2}\right) \operatorname{sen}(\theta) \\
z=R \cos \left(\phi_{2}\right)
\end{gathered}
$$

Na Fig. 6 podemos observar que $\phi_{1}$ é a latitude e $\theta$ é a longitude do ponto $P$.

Na Fig. 7 podemos observar que o triângulo retângulo OPQ nos fornece a equação (10) onde $R$ é a hipotenusa e $z$ é o cateto oposto ao ângulo $\phi_{1}$ :

$$
\phi_{1}=\operatorname{arcsen}(z / R)
$$

Da equação (9) obtemos a relação:

$$
\cos ^{2}\left(\phi_{2}\right)=\frac{z^{2}}{R^{2}}
$$

A partir do teorema fundamental da trigonometria temos:

$$
\operatorname{sen}^{2}\left(\phi_{2}\right)=1-\cos ^{2}\left(\phi_{2}\right)
$$

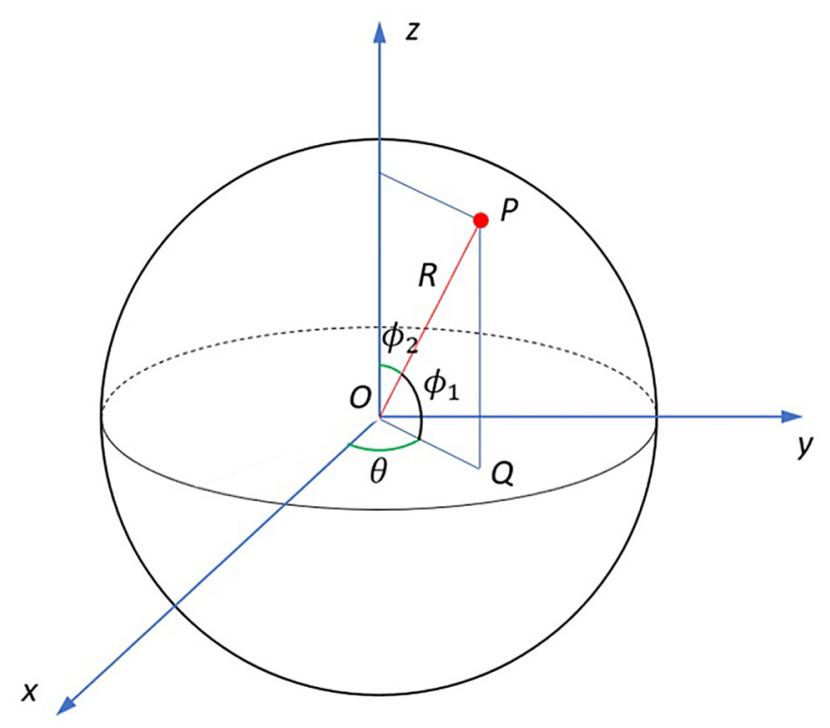

Figura 6: Modelo de aproximação para Esfera lunar.

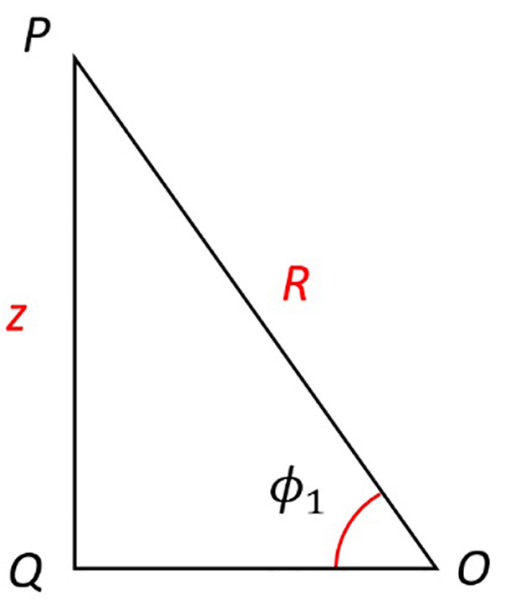

Figura 7: Triângulo retângulo OPQ. Fonte: Elaborado pelos autores.

Substituindo (12) em (11), teremos:

$$
\operatorname{sen}\left(\phi_{2}\right)=\frac{1}{R} \sqrt{R^{2}-z^{2}}
$$

Substituindo a equação (13) na equação (8) obtemos:

$$
y=\sqrt{R^{2}-z^{2}} \operatorname{sen}(\theta)
$$

Que resulta em:

$$
\theta=\operatorname{arcsen}\left(\frac{y}{\sqrt{R^{2}-z^{2}}}\right)
$$

Um resultado comparativo pode ser observado quando os dados obtidos através do software T̂asy são analisados juntamente com valores de medições executadas por observatórios astronômicos, como veremos a seguir.

\section{Resultados e Discussões}

A partir dos dados coletados construímos uma planilha com o objetivo de analisar os dados obtidos e verificar a precisão do nosso software como ferramenta para determinação dos diâmetros de crateras lunares, bem como para o estudo selenográfico de posição e relevo na superfície da Lua. Abaixo apresentamos as informações obtidas através de medições feitas pelo $\hat{I}$ asy e por dados conhecidos atualmente na literatura.

A Tab. 1 mostra os diâmetros conhecidos das crateras da Lua, segundo valores do Observatório Lunar Vaz Tolentino [14], e os valores obtidos por meio do programa Íasy. A Tab. 2 apresenta as diferenças entre os dados das coordenadas de latitude e longitude adquiridas no programa Iasy em comparação novamente com as informações extraídas do Observatório Lunar Vaz Tolentino [14].

Analisando os resultados das tabelas, fica evidente que o software $\hat{I}$ asy apresenta resultados próximos aos valores de referência, uma vez que a maioria dos erros 
Tabela 1: Comparação entre os diâmetros conhecidos de crateras lunares e os obtidos pelo software lasy. Fonte: http://www.vaztolentino.com.br.

\begin{tabular}{lccc}
\hline Cratera & Diâmetro conhecido* $(\mathrm{km})$ & Diâmetros obtidos com o Îasy $(\mathrm{km})$ & Diferença percentual \\
\hline Posidonius & 101 & 101,8 & $+0,8 \%$ \\
Catharina & 104 & 102,9 & $-1,1 \%$ \\
Hercules & 71 & 69,5 & $-2,1 \%$ \\
Atlas & 87 & 86,9 & $-0,1 \%$ \\
Eudoxus & 67 & 67,1 & $+0,1 \%$ \\
Aristóteles & 87 & 89,4 & $+2,8 \%$ \\
Theophilus & 100 & 101,8 & $+1,8 \%$ \\
Copernicus & 96 & 94,4 & $-1,7 \%$ \\
Kepler & 29 & 29,8 & $+2,8 \%$ \\
Plato & 101 & 99,3 & $-1,7 \%$ \\
Aristarchus & 40 & 39,7 & $-0,8 \%$ \\
Tycho & 85 & 86,9 & $+2,2 \%$ \\
Archimedes & 81 & 81,9 & $+1,1 \%$ \\
\hline
\end{tabular}

Tabela 2: Comparação entre os dados obtidos através do lasy e os dados conhecidos sobre a localização de algumas crateras lunares. Fonte: http://www.vaztolentino.com.br.

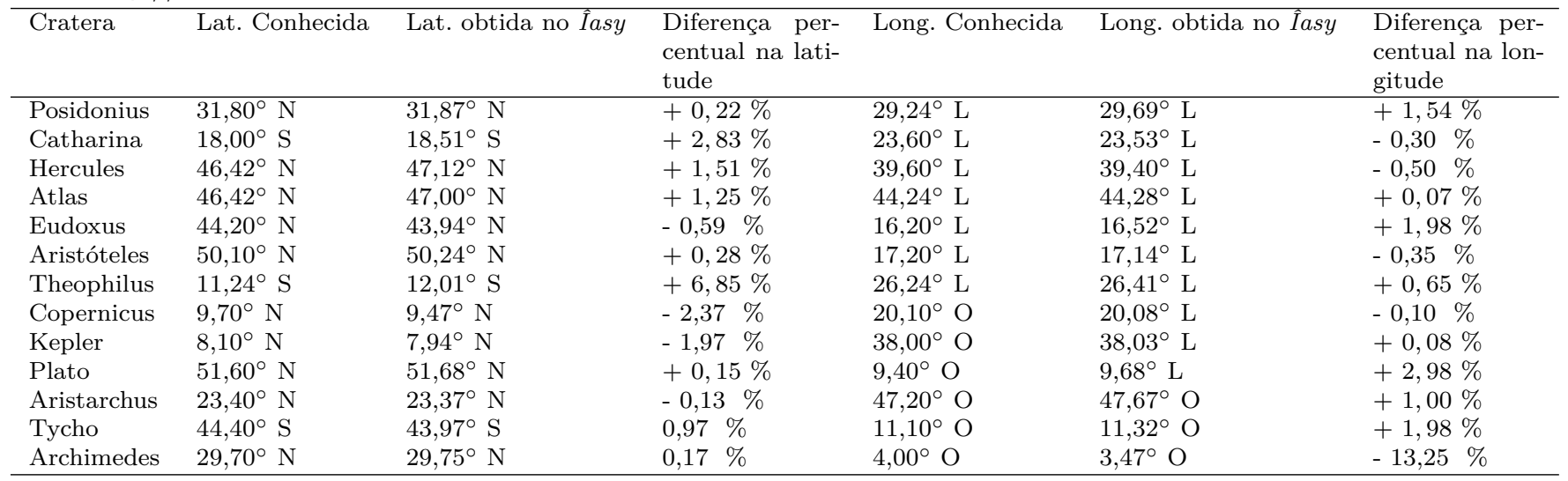

percentuais é menor que $3 \%$. Dependendo da forma como o usuário do software promove suas medições a precisão do programa será mais ou menos significativa. Assim, uma vez observados os parâmetros de ajuste das elipses de contorno das crateras e a escolha dos pontos que definem a circunferência ajustável ao disco lunar, é possível que os resultados destas medidas se tornem mais acurados. Sendo assim, consideramos nossas estimativas como uma boa aproximação, embora tal precisão não seja absolutamente necessária se os objetivos do usuário estiverem voltados apenas para fins educacionais ou de divulgação científica.

Acreditamos que a utilização do software $\hat{I} a s y$ como ferramenta de apoio ao ensino pode facilitar o entendimento e compreensão de conceitos matemáticos abstratos. Segundo a BNCC, dentre as habilidades específicas para o ensino de física destacam-se:

- Elaborar explicações, previsões e cálculos a respeito dos movimentos de objetos na Terra, no Sistema Solar e no Universo com base na análise das interações gravitacionais, com ou sem o uso de dispositivos e aplicativos digitais (como softwares de simulação e de realidade virtual, entre outros).
- Interpretar resultados e realizar previsões sobre atividades experimentais, fenômenos naturais e processos tecnológicos, com base nas noções de probabilidade e incerteza, reconhecendo os limites explicativos das ciências.

- Construir questões, elaborar hipóteses, previsões e estimativas, empregar instrumentos de medição e representar e interpretar modelos explicativos, dados e/ou resultados experimentais para construir, avaliar e justificar conclusões no enfrentamento de situaçõesproblema sob uma perspectiva científica. [6].

Uma forma de fazer isso é propor o ensino de geometria articulado a utilização do programa Îasy. O professor pode trabalhar as definições de inclinação da reta, mediatriz, triângulos, circunferência e elipse, e utilizar o Îasy como ilustração. Outros conteúdos possíveis de serem abordados são os conceitos de trigonometria esférica, trigonometria no triângulo retângulo e transformação de coordenadas, para mostrar como é possível transformar coordenadas retangulares em coordenadas esféricas. Ainda pode-se realizar o estudo de escalas, já que o programa transforma os dados de pixels para quilômetros, uma vez determinado o raio da Lua como base. 
Dentro das competências de investigação e compreensão dos fenômenos físicos, como estabelecido pela BNCC, é possível, como forma de investigação científica, sugerir aos estudantes que estes determinem os tamanhos e localizações de crateras da Lua e de outros corpos celestes. Embora o ÎIsy tenha sido construído para trabalhar com crateras da Lua, é possível utilizá-lo para estudar crateras de outros lugares. O professor pode propor uma atividade de investigação, cujo objetivo será determinar o tamanho de crateras na Lua, nas luas de Júpiter, nas crateras de planetas como Mercúrio, Vênus, Marte etc., ou de crateras em asteroides com formatos esféricos. Além disso, não é preciso se limitar apenas a crateras. Basicamente, qualquer estrutura circular ou elíptica visível na superfície de corpos celestes pode ser dimensionada pelo $\hat{I}$ asy. É o caso, por exemplo, das tempestades no planeta Júpiter, dos continentes e mares lunares, das calotas polares de Marte ou da própria Terra, de grandes tempestades na Terra que possam ser visualizadas do espaço, das manchas solares etc.

\section{Considerações Finais}

Em virtude da precisão dos resultados obtidos, podemos dizer que o programa Íasy apresenta resultados satisfatórios na determinação das dimensões das crateras lunares, assim como, na determinação das coordenadas selenográficas dessas crateras. Além disso, o software desenvolvido na plataforma Microsoft Visual Basic tem a importante característica de utilizar um conjunto de equações matemáticas relativamente simples (algumas delas ocasionalmente utilizadas no Ensino Médio), o que torna seu algoritmo bastante acessível. O software $\hat{I} a s y$ está disponível para download na página do Clube de Astronomia VEGA, do IFPE - campus Pesqueira, no link vega.pesqueira.ifpe.edu.br.

Considerando que as atividades científicas envolvendo Astronomia, realizadas em espaços não escolares de ensino, podem apresentar um grande potencial para a aprendizagem [9], o programa T̂sy pode ser utilizado também como ferramenta nos ensinos (escolar ou não-escolar) de física, matemática, geografia, informática, entre outros. Assim, diante da praticidade e multidisciplinaridade ele pode servir, inclusive, como uma ferramenta de instrumentação capaz de despertar a curiosidade científica de estudantes nas mais diversas modalidades e níveis.

Como objeto de investigação, o software desenvolvido também pode ser relevante nas práticas da Astronomia amadora como ferramenta de iniciação e pesquisa científica.

\section{Referências}

[1] S.T. Heath, Aristarchus of Samos, the Ancient Copernicus (Oxford University Press, Cambridge, 1913).

[2] H.M. Nussenzveig, Curso de física básica: mecânica (Editora Blucher, São Paulo, 2013).
[3] G. Galilei, Sidereus Nuncius: O Mensageiro das Estrelas (Fundação Calouste Gulbenkian, Lisboa, 2015).

[4] R.L. Wildey, Icarus 3, 136 (1964).

[5] T. Kneissl, S. Van Gasselt e G. Neukum, Planetary and Space Science 59, 1243 (2011)

[6] MINISTÉRIO DA EDUCAÇÃO. Base nacional comum curricular, disponível em: http://basenacionalcomum.mec.gov.br/.

[7] R. Langhi, Um estudo exploratório para a inserção da Astronomia na formação de professores dos anos iniciais do Ensino Fundamental. Dissertação de Mestrado, Universidade Estadual Paulista, São Paulo (2004).

[8] R. Scott, X. Shen, I. Mulley e Z. Pan, Physics Education 48, 520 (2013)

[9] R, Langhi e B.A. Martins, Caderno Brasileiro de Ensino de Física 35, 64 (2018).

[10] K. Joy, I. Crawford, P. Grindrod, C. Lintott, S. Bamford, A. Smith, A. Cook, I. Antonenko, R. Balfanz, M. Balme et al, Astronomy \& Geophysics 52, 10 (2011).

[11] E.E. Prather, S. Cormier, C.S. Wallace, C. Lintott, M.J. Raddick e A. Smith, Astronomy Education Review 12, 010109 (2013).

[12] INSTITUTO DE FÍSICA DA UFRGS, Parâmetros físicos e astronômicos, disponível em: http://astro.if ufrgs.br/dados.htm

[13] D.M.B. Costa, J.L. Teixeira, P.H. Siqueira e L.V. SOUZA, Elementos de geometria: geometria plana e espacial (Universidade Federal do Paraná, Curitiba, 2012) $3^{\circ} \mathrm{ed}$.

[14] Vaz Tolentino Observatório Lunar, disponível em: http: //vaztolentino.com.br, acessado em 06/11/2019. 